\title{
The unnatural order of things
}

\author{
The ability of DNA to self-assemble into a variety of nanostructures and nanomachines is being \\ exploited by a growing number of researchers.
}

Although it is difficult to overstate the importance of DNA in biology, this famous molecule is also becoming more prominent in nanotechnology. The appeal of DNA to nanoscientists is three-fold: first, it is a natural nanoscale material; second, a large number of techniques for studying DNA are already available; and third, its ability to carry information (which is its main role in biology) can be exploited in the selfassembly process. All this can be seen by a number of articles in this and upcoming issues of Nature Nanotechnology ${ }^{1}$.

DNA molecules comprise a backbone of repeated sugar-phosphate units, with one of the four bases - adenine (A), cytosine $(C)$, guanine $(G)$ or thymine (T) - attached to each sugar. The twisting ladder of the double helix is formed by combining (or hybridizing) two antiparallel DNA strands, which are held together by hydrogen bonds between the complementary bases: adenine to thymine $(\mathrm{A}-\mathrm{T})$ and cytosine to guanine $(\mathrm{C}-\mathrm{G})$. By exploiting these exquisite base-pairing rules, which provide DNA with its ability to pass genetic information from generation to generation, self-assembled structures can be built simply by programming sequences of DNA - jigsaw pieces for one-, two- or three-dimensional puzzles. This process, combined with the current ability to synthesize almost any sequence in an automated fashion, means that it is possible to make new structures and devices that are not found in nature ${ }^{2}$.

A typical building block, or tile, in structural DNA nanotechnology has a rigid core with 'sticky' ends: single strands of DNA that act like a smart glue to link the double-helical regions together ${ }^{2}$. A diverse range of tiles have already been devised and the construction principles continue to push the boundaries of what can be designed and built ${ }^{3,4}$. One of the most intriguing recent advances is DNA origami ${ }^{5,6}$, in which a lengthy single strand of DNA is folded into a shape using a number of shorter 'stapling' strands. The result is an approach that can create large, complex and eye-catching nanostructures with relative ease ${ }^{6}$.

But these designs may have function as well as form. By incorporating binding sites at selected positions, DNA nanostructures can be used to precisely organize other species, such as proteins or nanoparticles ${ }^{7}$. This process of positioning individual species on DNA 'scaffolds' has now moved on to making the species work together. On page 249 Itamar Willner and colleagues describe how the organizational skills of DNA can be used to attach two different enzymes (or an enzyme and its cofactor) to the scaffold at specific positions to activate a cascade of enzyme reactions. In the absence of the scaffold similar enzymatic reactions could not occur. The positioning of the enzyme on the scaffold could also be used to control the reactivity (see also page 211 ).

\section{DNA is one of the most promising candidates for molecular computing.}

Elsewhere, on page 245, Ned Seeman and colleagues report a dynamic form of patterning using DNA tiles. They show that a target molecule, which can be in a variety of shapes including triangles and diamonds, can be captured between two independently programmed DNA devices. The DNA devices are based on a DNA molecule that can be switched between two different states that differ by a halfrotation of one end relative to the other. These devices are themselves placed on a substrate built from DNA origami tiles and they can be programmed to capture a specific molecule before or after they are bound to the origami array. The system incorporates an error-correction procedure, where carefully controlled heating allows any incorrectly captured molecules to be released and replaced by the targeted species; a procedure that returns flawless results every time.

Synthetic machinery can also be constructed using $\mathrm{DNA}^{8}$. These nanomachines can be bipedal walkers that run along self-assembled tracks or autonomous motors that obtain energy by catalysing the reaction of DNA fuels. The simplest of active structures are switches or actuators that move between two states in response to a molecular or environmental trigger. It has been suggested that certain devices have potential uses in medicine, but artificial DNA devices have until recently been limited to in vitro applications. Now, Yamuna Krishnan and colleagues have shown that a DNA nanomachine can function inside living cells ${ }^{9}$. The device, called an I-switch, is triggered by protons and acts as a pH sensor inside the cell.

Alternatively, and as Richard Jones points outs on page 207, DNA is one of the most promising candidates for molecular computing. Base-pair interactions have, for example, been used to design basic logic circuits, including AND, OR and NOT gates ${ }^{10}$. Or, in a more disorderly approach to organization, DNA can aid the selfassembly of nanoparticles. For example, DNA strands randomly scattered around a gold nanoparticle can be used to create three-dimensional crystalline assemblies ${ }^{11,12}$. The DNA strands are covalently bonded to the gold by means of a sulphur atom attached to one end of the strand. By mixing nanoparticles capped with two different types of strands, the free ends of which are complementary and capable of forming bridges, the nanoparticles can organize themselves into ordered arrays. The arrays can have a body-centred-cubic crystal symmetry, with the different capped nanoparticles taking alternating positions in the lattice.

Significant challenges lie ahead if practical materials are to emerge from DNA nanotechnology, such as the scalingup of structures ${ }^{3,7}$, or the continued progression to three dimensions ${ }^{4}$. But DNA has already proven to be an excellent construction material, and the field of DNA nanotechnology is just starting out in life. $\square$

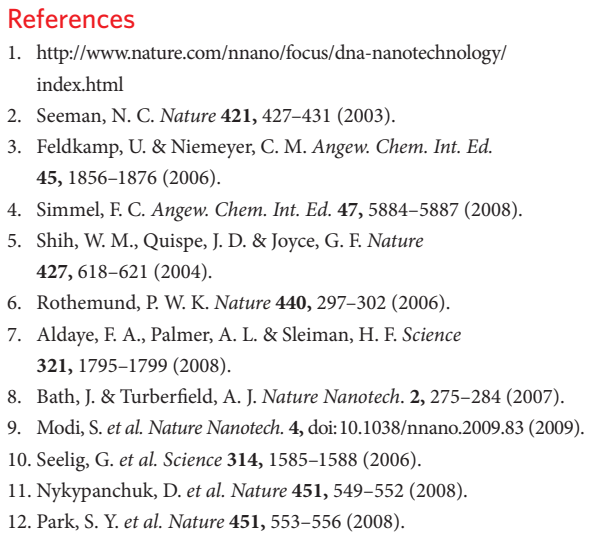

